\title{
Nurses' Voices: Autumn 2020
}

\author{
Kim, Jeung-Im \\ College of Medicine, School of Nursing, Soonchunhyang University, Cheonan, Korea
}

What are the kinds of research papers not cited by studies in international journals? Does not being cited make these papers not valuable?

This point is especially crucial in the evaluation of a journal. Hence, commencing with this issue, we are starting an editorial section to improve the impact factor of our journal, including the number of citations to it. Such an editorial can aid readers to find many valuable papers. It can also make readers realize the significance of the papers being published and help to draw specific directions for future studies.

Studies on working nurses continue to be carried out. This can be gleaned from a report by the Korean Citation Index (KCI) [1]. The KCI web word cloud on October 15, 2020 showed the top 10 study keywords to be knowledge, validation study, nurses, nursing psychometrics, diabetes, self-efficacy, nursing staff, simulation training, and workplace [1]. Several researchers in generalhave dealt with workplace bullying after they perceived the severity of bullying in their workplace after 2013. Society appeared to become visibly concerned about nurses when one nurse in Seoul died on January 5, 2019 over "heavy workload", and "bullying" [2], but this concern appeared to last for a short period, and such an accident did not lead to significant changes in the working environment, especially in hospitals. In this issue, several researchers dealt with nurses. One reported on the quality of sleep and related factors of novice and experienced nurses working with a three-shift system. Surprisingly, sleep quality was lower among the experienced nurses than among the novice nurses. Furthermore, the two factors of sleep hygiene and fatigue were identified to have affected the sleep quality of the nurses ("Secondary Data Analysis on the Quality of Sleep and Related Factors of Novice and Experienced Shift Work Nurses") [3]. Considering these findings, there is a need to identify why the experienced nurses experienced lower sleep quality than novice nurses.

Another study investigated work violence and turnover intention among hospital nurses. Through this study, it was shown that many nurses experience violence in the course of carrying out their duties, such as having their personality and professional status attacked, falling victim to bullying due to work, as well as experiencing direct physical attacks. Such violence directly influences turnover intention. Furthermore, the mediator for turnover intention after controlling for the general characteristics of marital status, work department, educational level, age, and clinical career, was identified to be resilience ("Relationship of Workplace Violence to Turnover Intention in Hospital Nurses: Resilience as a Mediator") [4].

One does not necessarily "provide resilience." You can provide measures that would lead to resilience, but a person is ultimately the source of his or her own resilience. How can we help to build nurses' resilience? When can we speak-out for the welfare of nurses? 'You can help at this moment by citing this issue'.

To address concerns or problems, one should have compre-

\section{Address reprint requests to : Kim, Jeung-Im}

College of Medicine, School of Nursing, Soonchunhyang University, 31 Soonchunhyang-ro 6-gil, Dongnam-gu, Cheonan 31151, Korea

Tel: +82-41-570-2493 Fax: +82-41-570-2498 E-mail: jeungim@sch. ac. kr

Received: October 16, 2020 Revised: October 16, 2020 Accepted: October 19, 2020 Published online October 31, 2020

This is an Open Access article distributed under the terms of the Creative Commons Attribution NoDerivs License. (http://creativecommons. org/licenses/by-nd/4. 0)

If the original work is properly cited and retained without any modification or reproduction, it can be used and re-distributed in any format and medium. 
hensive knowledge about the issues at hand, and to acquire knowledge, read and cite the Journal of Korean Academy of Nursing (JKAN), an international journal-coming to you in beautiful autumn.

\section{CONFLICTS OF INTEREST}

The authors declared no conflict of interest.

\section{REFERENCES}

1. Korea Citation Index. [Internet]. Daejeon. [Cited in 2020 Oct 15]. Available from https://www.kci.go.kr/kciportal/po/search/ poCitaView. kci $?$ sereId $=000229 \&$ from $=$ sereDetail .

2. The Korea Times. [Internet]. Seoul. [Cited in Oct 20]. Available from https://www.koreatimes.co.kr/www/nation/2019/09/ 119_275206.html.

3. Yu M, Choi-Kwon S. Secondary Data Analysis on the Quality of Sleep and Related Factors of Novice and Experienced Shift Work Nurses. Journal of Korean Academy of Nursing. 2020;50;e7.

4. Relationship of Workplace Violence to Turnover Intention in Hospital Nurses: Resilience as a Mediator. Journal of Korean Academy of Nursing. 2020. Forthcoming. 